Published in final edited form as:

Clin Cancer Res. 2018 April 15; 24(8): 1932-1943. doi:10.1158/1078-0432.CCR-17-1928.

\title{
Ponatinib shows potent antitumor activity in small cell carcinoma of the ovary hypercalcemic type (SCCOHT) through multi-kinase inhibition
}

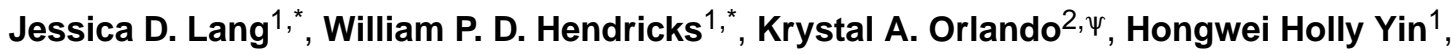 \\ Jeffrey Kiefer ${ }^{1}$, Pilar Ramos ${ }^{1}$, Ritin Sharma ${ }^{3}$, Patrick Pirrotte ${ }^{3}$, Elizabeth A Raupach ${ }^{1,3}$, \\ Chris Sereduk $^{1}$, Nanyun Tang ${ }^{1}$, Winnie Liang ${ }^{1}$, Megan Washington ${ }^{1}$, Salvatore J. Facista ${ }^{1}$, \\ Victoria L. Zismann ${ }^{1}$, Emily M. Cousins ${ }^{4}$, Michael B. Major ${ }^{4}$, Yemin Wang ${ }^{5}$, Anthony N. \\ Karnezis $^{5}$, Aleksandar Sekulic ${ }^{1,6}$, Ralf Hass ${ }^{7}$, Barbara Vanderhyden ${ }^{8}$, Kesavannair \\ Praveen $^{9}$, Bernard E. Weissman ${ }^{2}$, David G. Huntsman ${ }^{5,10}$, and Jeffrey M. Trent ${ }^{1}$ \\ ${ }^{1}$ Division of Integrated Cancer Genomics, Translational Genomics Research Institute (TGen), \\ Phoenix, AZ, USA. \\ ${ }^{2}$ Department of Pathology and Laboratory Medicine, Lineberger Cancer Center, University of \\ North Carolina, Chapel Hill, NC, USA. \\ ${ }^{3}$ Collaborative Center for Translational Mass Spectrometry, Translational Genomics Research \\ Institute (TGen), Phoenix, AZ, USA. \\ ${ }^{4}$ Department of Cell Biology and Physiology, Lineberger Cancer Center, University of North \\ Carolina, Chapel Hill, NC, USA. \\ ${ }^{5}$ Department of Pathology and Laboratory Medicine, University of British Columbia and \\ Department of Molecular Oncology, British Columbia Cancer Research Centre, Vancouver, BC, \\ Canada. \\ ${ }^{6}$ Department of Dermatology, Mayo Clinic, Scottsdale, AZ, USA. \\ ${ }^{7}$ Department of Obstetrics and Gynecology, Hannover Medical School, D-30625 Hannover, \\ Germany.
}

${ }^{8}$ Department of Cellular and Molecular Medicine, University of Ottawa and Cancer Therapeutics Program, Ottawa Hospital Research Institute, Ottawa, ON, Canada.

${ }^{9}$ Molecular Response, San Diego, CA, USA.

${ }^{10}$ Department of Obstetrics and Gynaecology, University of British Columbia, Vancouver, BC, Canada.

\section{Structured Abstract}

\footnotetext{
Corresponding Author: Jeffrey M Trent, 445 N $5^{\text {th }}$ St. Phoenix, AZ 85004, Phone: (602) 343-8419, Fax: (602) 343-8448, jtrent@tgen.org.

These authors contributed equally to this work.

$\Psi$ Authorship change since initial submission. Author was moved up in author list to reflect the significant contributions she has made to the manuscript revisions in generating and optimizing the COV434 inducible cell line

COI disclosure statement: The authors declare no potential conflicts of interest.
} 
Purpose: Small cell carcinoma of the ovary, hypercalcemic type (SCCOHT) is a rare, aggressive ovarian cancer in young women that is universally driven by loss of the SWI/SNF ATPase subunits SMARCA4 and SMARCA2. A great need exists for effective targeted therapies for SCCOHT.

Experimental Design: To identify underlying therapeutic vulnerabilities in SCCOHT, we conducted high-throughput siRNA and drug screens. Complementary proteomics approaches profiled kinases inhibited by ponatinib. Ponatinib was tested for efficacy in two patient-derived xenograft (PDX) models and one cell line xenograft model of SCCOHT.

Results: The receptor tyrosine kinase (RTK) family was enriched in siRNA screen hits with FGFRs and PDGFRs being overlapping hits between drug and siRNA screens. Of multiple potent drug classes in SCCOHT cell lines, RTK inhibitors were only one of two classes with selectivity in SCCOHT relative to three SWI/SNF-wild-type ovarian cancer cell lines. We further identified ponatinib as the most effective clinically approved RTK inhibitor. Re-expression of SMARCA4 was shown to confer a 1.7 -fold increase in resistance to ponatinib. Subsequent proteomic assessment of ponatinib target modulation in SCCOHT cell models confirmed inhibition of nine known ponatinib target kinases alongside 77 non-canonical ponatinib targets in SCCOHT. Finally, ponatinib delayed tumor doubling time four-fold in SCCOHT-1 xenografts while reducing final tumor volumes in SCCOHT PDX models by $58.6 \%$ and $42.5 \%$.

Conclusion: Ponatinib is an effective agent for SMARCA4-mutant SCCOHT in both in vitro and in vivo preclinical models through its inhibition of multiple kinases. Clinical investigation of this FDA-approved oncology drug in SCCOHT is warranted.

\section{Keywords}

SCCOHT; SWI/SNF; ponatinib; SMARCA4; SMARCA2

\section{Introduction}

Small cell carcinoma of the ovary, hypercalcemic type (SCCOHT), a rare and aggressive form of ovarian cancer, is diagnosed in women at a median age of 24 years (range: 14 months to 47 years) (1). Meta-analysis of 257 clinically annotated SCCOHT cases has shown a dismal two-year survival rate less than $35 \%$. The most effective treatment regimen based on this assessment is surgery followed by aggressive high dose chemotherapy, radiation, and stem cell rescue $(1,2)$. The poor response rates and extreme toxicity of this regimen necessitate identification of effective, targeted treatments for these young patients. SCCOHTs are characterized by inactivating germline and somatic mutations in the tumor suppressor $S M A R C A 4$ (also known as BRG1) resulting in concomitant protein loss in nearly all cases (3-8). These $S M A R C A 4$ alterations occur amidst otherwise diploid SCCOHT genomes and very rare secondary mutations in other cancer genes $(4,8)$. SMARCA4 is one of two mutually exclusive ATPase subunits of the SWI/SNF chromatinremodeling complex that plays a central role in regulation of transcriptional programs associated with differentiation. The alternative SWI/SNF ATPase, SMARCA2 (also known as BRM), is also absent in SCCOHT due to epigenetic silencing $(3,7)$. Thus, these tumors are driven by a unique genotype that fuels broad transcriptional dysregulation through SWI/SNF dysfunction. 
Several other tumor types are also universally characterized by inactivation of SWI/SNF complex members including thoracic sarcomas bearing SMARCA4 mutation and SMARCA2 loss (9), rhabdoid tumors which are universally characterized by SMARCB1 (also known as SNF5) mutations alongside SMARCA2 silencing and expression loss in 70\% of cases $(10,11)$, and renal medullary cancers also characterized by SMARCB1 loss $(12,13)$. Other cancers with a significant proportion of SWI/SNF mutations include ovarian clear cell carcinomas and endometrioid carcinomas ( $\sim 50 \%$ and $\sim 30 \%$ with ARID1A loss, respectively) $(14,15)$ and non-small cell lung cancers $(\sim 10 \%$ of primary tumors with dual SMARCA4 and SMARCA2 loss) $(16,17)$. Overall, an estimated $20 \%$ of human cancers bear potentially oncogenic mutations in one or more SWI/SNF complex subunits $(18,19)$. Thus, identification of therapeutic vulnerabilities in SWI/SNF-mutant cancers with relatively simple genomes such as SCCOHT may hold broader relevance for more diverse cancers.

Preclinical studies to date have suggested that several experimental agents such as foretinib (c-Met inhibitor), epothilone B (tubulin inhibitor), or oncolytic viruses may be effective in SCCOHT (20-22). We and others have also shown that investigational epigenetic agents such as bromodomain and EZH2 inhibitors may hold promise for treatment of these cancers (23-25). Yet, despite the prevalence of pathogenic SWI/SNF mutations in cancer, no approved targeted cancer drugs have yet shown activity in the setting of loss of these tumor suppressors. In order to identify novel therapeutic vulnerabilities conferred by SWI/SNF dysfunction in SCCOHT with a focus on identification of targeted FDA-approved oncology drugs, we performed high-throughput (HT) siRNA and drug screens in SCCOHT cell lines. We identified dependence on receptor tyrosine kinase (RTK) signaling both via enrichment for RTK hits in the siRNA screen as well as discovery of SCCOHT hypersensitivity to the class-representative RTK inhibitor (RTKi) PD-161570 in the chemical screen. Of drug screen hits by class, only RTK inhibitors (PD-161570) and metal chelators (ammonium pyrrolidinedithiocarbamate) were selective hits in SMARCA4-mutant SCCOHT cells relative to three SWI/SNF-wild-type ovarian cancer cell lines. Subsequent evaluation of a panel of FGFR/PDGFR-selective RTK inhibitors highlighted ponatinib as the most potent tested agent in SCCOHT cell lines, and an inducible SMARCA4 SCCOHT cell line demonstrates that restoration of SMARCA4 expression reduces the sensitivity of SCCOHT cells to ponatinib. These observations align with prior data in SWI/SNF-mutant rhabdoid tumor models in which expression of the ponatinib target FGFR was shown to be associated with SMARCB1 and SMARCA4 loss, conveying sensitivity to RTK inhibitors including ponatinib (26,27). Further, activation of downstream AKT was shown to be elevated in SMARCB1-deficient tumor models $(28,29)$ and dual inhibition of PDGFRa and FGFR1 in MRT cell lines was more effective than specific inhibitors (30). Here, we additionally show via proteomic assessment of target engagement and downstream signaling that ponatinib's effects in SCCOHT are also mediated by dependence on signaling of multiple RTKs. Finally, we show the ability of ponatinib to significantly delay tumor progression in a cell line xenograft model of SCCOHT and markedly reduce tumor growth of patient-derived xenograft (PDX) models of SCCOHT, thereby prioritizing ponatinib for a clinical trial in SCCOHT patients. 


\section{Materials and Methods}

\section{Cell lines}

BIN67, and SCCOHT-1 (SCCOHT), G401 and G402 (MRT), H522 (lung adenocarcinoma), and OVCAR-3, OVCAR-5, and OVCAR-8 (SWI/SNF-wildtype high-grade serous ovarian cancer) cells were maintained in RPMI 1640 (Thermo Fisher Scientific, Waltham, MA, USA) supplemented with 10\% Fetal Bovine Serum (FBS; Thermo Fisher Scientific) and 1\% Penicillin/Streptomycin (Thermo Fisher Scientific). COV434 cells, previously identified as derived from a juvenile granulosa cell tumor have now been re-categorized as SCCOHT based on SMARCA4 mutation and lack of SMARCA2 expression (24). A427 (lung adenocarcinoma) and HepG2 (hepatocellular carcinoma) cells were maintained in EMEM (Thermo Fisher Scientific) supplemented with 10\% FBS and 1\% Penicillin/Streptomycin. COV434 (SCCOHT) cells were maintained in DMEM (Thermo Fisher Scientific) supplemented with 10\% FBS and 1\% Penicillin/Streptomycin. SVOG3e (SV40-transformed ovarian cells) cells were maintained in DMEM/F12 (Thermo Fisher Scientific) supplemented with $10 \%$ FBS and $1 \%$ Penicillin/Streptomycin. All cells were maintained at $37^{\circ} \mathrm{C}$ in a humidified incubator containing $5 \% \mathrm{CO}_{2}$. All cell lines were routinely monitored for mycoplasma testing and STR profiled for cell line verification.

Inducible SMARCA4 COV434 cells (COV434 pIND20 BRG1-2.1 and 2.7) were established by lentiviral infection of COV434 cells with pInducer20-Brg1. Resulting G418resistant clones were further subcloned and screened for doxycycline-inducible SMARCA4. Optimization of SMARCA4 induction by doxycycline is shown in Supplemental Figure 1.

\section{Western blotting}

Whole-cell extracts from cell lines were prepared using RIPA buffer (Santa Cruz Biotechnology, Dallas, TX, USA) with protease and phosphatase inhibitors using standard protocols. Thirty $\mu \mathrm{g}$ protein was loaded per well on NuPage 4-12\% Bis-Tris gels and subsequently transferred to PVDF membranes. Blots were pre-blocked in 5\% non-fat dry milk in TBST or BSA in TBST for 1 hour, and probed using primary antibody overnight. Blots were incubated with secondary antibody (anti-rabbit IgG-HRP, Cell Signaling Technology, Danvers, MA, USA; anti-mouse IgG-HRP, Santa Cruz Biotechnology) at 1:5000 for 2 hours and developed using Pierce ECL Western Blotting Substrate or SuperSignal West Femto Substrate (Thermo Fisher Scientific). Primary antibodies (Cell Signaling Technology): phospho-p38, total p38, phospho-Akt T308, phospho-Akt S473, total Akt, GAPDH.

\section{Phospho-RTK profiling}

For cell lines, BIN67 and SCCOHT-1 cells were treated with ponatinib at $\mathrm{IC}_{30}, \mathrm{IC}_{50}$, and $1 \mu \mathrm{M}$ for 1 hour. For pharmacodynamic studies on PDX tumor tissues, flash-frozen tumors following a six hour treatment with ponatinib or tumors collected at the efficacy study endpoint ( $>30$ days) were used. Phospho-RTK profiling was performed using the Proteome Profiler Human Phospho-RTK Array Kit (R\&D Systems, Minneapolis, MN, USA) according to manufacturer's recommendations. Briefly, cells were lysed and the BCA assay was performed to quantify protein concentration. Three hundred $\mu \mathrm{g}$ of lysate was incubated 
on each blot overnight, and bound phosphorylated protein was detected using HRPconjugated anti-phospho-tyrosine antibody. Arrays were developed using Pierce ECL Western Blotting Substrate (Thermo Fisher Scientific), and images were quantified using ImageJ software (NIH) using built-in gel quantification and background subtraction tools. Duplicate spots were averaged for each RTK, and ponatinib-treated samples were normalized to vehicle-treated samples.

\section{Animal studies}

All procedures were carried out under the institutional guidelines of TGen Drug Development's Institutional Animal Care and Use Committee or the Animal Care Committee of the University of British Columbia (SCCOHT-1 model, A14-0290). For SCCOHT-1 xenograft experiments, $1 \times 10^{7}$ cells in 50\% Matrigel / 50\% Media (Corning) in a final volume of $200 \mu \mathrm{l}$ were subcutaneously inoculated into the backs of NRG (NOD.CgRag $1^{\mathrm{tm} 1 \mathrm{Mom}} \mathrm{Il} 2 \mathrm{rg}^{\mathrm{tm} 1 \mathrm{Wjl} / \mathrm{SzJ})}$ mice. For PDX experiments, histologically confirmed SMARCA4-mutant SCCOHT tumors PDX-465 and PDX-040 were acquired from Molecular Response (MRL, San Diego, CA, USA) and serially passaged in mice (3). Tumor suspension in 50\% Matrigel / 50\% Media in a final volume of $100 \mu \mathrm{L}$ were subcutaneously inoculated into the backs of NOG (NOD.Cg-Prkdc ${ }^{\text {scid }}$ Il2rg ${ }^{\text {tm } 1 \text { Sug } / J i c T a c) ~ m i c e . ~ M i c e ~ w e r e ~}$ randomized to treatment arms $(\mathrm{n}=8)$ once the average tumor volume reached $100-400 \mathrm{~mm}^{3}$ (SCCOHT-1 model) or 75-125 mm³ (PDX models). Ponatinib (Selleckchem, Houston, TX, USA or Activebiochem (SCCOHT-1)) was formulated in $25 \mathrm{mM}$ citrate buffer (pH 2.75). Vehicle or ponatinib (15 or $30 \mathrm{mg} / \mathrm{kg}$ for SCCOHT-1 or PDX models, respectively) was administered by oral gavage daily for 30 days or until humane endpoint (tumor reaches $1000 \mathrm{~mm}^{3}$ ). Tumor size and body weight were measured twice weekly until endpoint. Tumors were excised upon necropsy and either frozen or formalin-fixed/paraffin-embedded.

Chemical library, siRNA screens, ABPP, MIB-MS, and RNA-Seq methods are described in Supplemental Materials and Methods.

\section{Results}

\section{RTK dependency identified by an siRNA screen in the SCCOHT cell line, BIN67}

In an effort to identify genetic dependencies in SCCOHT, HT siRNA libraries were used to test necessity of clinically actionable genes or growth of the SCCOHT cell line BIN67. We transfected siRNAs targeting over 7,000 genes in both Druggable Genome (DGv3) and also custom kinome (vKINv4) libraries. Hits were determined based on measurement of cell viability reduction using CellTiter Glo measurements analyzed via a combination of redundant siRNA activity score (RSA) p-value ranking with ranking by mean signal intensity and utilizing a p-value cutoff of 0.05 (see Supplemental Materials and Methods for further detail). Of 246 genes from the DGv3 library and 81 genes from the vKINv4 library identified as hits based on these criteria, 109 were also validated through independent confirmation that two of four siRNAs inhibited viability by $>50 \%$ (See Supplemental Materials and Methods and Supplemental Table 1). 
The ReactomeFI Cytoscape plugin was used to identify pathways enriched in these validated hits (Table 1) (31). Top enriched gene sets were dominated by receptor tyrosine kinase (RTK) signaling, including FGFRs, PDGFRs, and EGFRs alongside the AKT and MAPK signaling cascades downstream of these RTKs. In the protein interaction network generated

from gene hits using ReactomeFI, RTK signaling similarly emerged as the central connected network, as demonstrated by the PDGFR signaling pathway (Figure 1A).

All kinase hits from the two BIN67 siRNA screens were also plotted on a kinome tree to identify key kinase families on which SCCOHT cells are reliant for growth (Supplemental Figure 2). Tyrosine kinases, particularly RTKs, were most strongly identified amongst the kinase hits. A second pathway analysis method, ClueGO, was used to visualize the biological concepts represented in the top hits (Supplemental Figure 3). This method also identified interaction between RTKs in top hits through functions in cancer signaling, regulation of actin cytoskeleton, and cell adhesion. Together, these data suggest a broad dependency of SCCOHT cells on RTK signaling networks.

\section{A high-throughput drug screen identifies selective potency of RTKi in SMARCA4-mutant SCCOHT cells relative to SWI/SNF wildtype ovarian cancers}

In an effort to identify novel candidate drugs for targeted treatment of SCCOHT in an unbiased manner, high-throughput (HT) drug screens were performed in BIN67 cells using the LOPAC and Prestwick libraries of a combined 2,300 small molecules. All of these compounds are pharmacologically active with well-annotated drug targets from a breadth of signaling pathways, and over half are approved for at least one clinical indication. We first identified compounds active in BIN67 SCCOHT cells, filtering out broadly cytotoxic drugs that also showed effect in HepG2 cells. Hits were defined as agents that reduced viability of BIN67 cells by $>50 \%$ relative to HepG2 according to CellTiter Glo measurements at 72 hours post-treatment (see Supplemental Materials and Methods for further detail). 64 compounds reduced growth of BIN67 but not HepG2 cells (Supplemental Table 2). Of these hits, 51 were next validated in 12-point drug dose-response (DDR) format in the SCCOHT cell lines BIN67, SCCOHT-1, and COV434 as well as a SMARCA4-wild-type human granulosa cell line SVOG3e and HepG2 cells. Of the 51 compounds tested, 42 were confirmed to have $\mathrm{IC}_{50}$ values $>10$-fold lower in BIN67 cells than HepG2 cells (Supplemental Table 3). Generally IC 50 values from BIN67 cells were similar to those in SCCOHT-1, COV434, and SVOG3e.

The 42 validated drug hits were annotated based on their reported targets in preclinical literature and vendor database annotations. This information was incorporated into a chemical-target/entity bipartite network and visualized using Cytoscape (Figure 1B). Network cliques with the highest number of connected compounds were associated with microtubule targeting or DNA damage induction. These network cliques are representative of current standard treatments for SCCOHT, which include vinblastine, doxorubicin, and etoposide (2). An additional clique identified was cardiac glycosides, which is an interesting finding and have not been used previously for SCCOHT. The chemical libraries also included $27 \mathrm{RTKi}$, of which nine were EGFR inhibitors, two were IGF-1R inhibitors, two were FGFR inhibitors, one was a PDGFR inhibitor, and the remaining targeted 
miscellaneous or unannotated RTKs. Two of these RTKi were identified as hits in the validation set: PD-161570 and Tyrphostin A9. While these agents are multi-RTK inhibitors, they have been shown to demonstrate some selectivity for FGFR1 and PDGFRs.

Next, to determine selectivity of these drug screen hits for SMARCA4-mutant SCCOHT among ovarian cancers, twenty drugs were chosen to represent the network cliques defined in Figure 1B and were further screened in three ovarian cancer cell lines lacking driver mutations in SWI/SNF genes (Figure 2C; portals.broadinstitute.org/ccle). Responses of OVCAR-3, OVCAR-5, and OVCAR-8 cells in DDR assays were tested. OVCAR-3 was, on average, more resistant the drugs, while OVCAR-5 and OVCAR- 8 showed similar sensitivities to most drugs as the SCCOHT cell lines. However, SCCOHT cell lines demonstrated unique sensitivity to only two drugs: PD-161570, an RTK inhibitor, and ammonium pyrrolidinedithiocarbamate, a metal chelator.

\section{Targeted RTKi screens prioritize ponatinib as an FDA-approved oncology drug with selective potency in SCCOHT cell lines}

To further explore RTK dependence in the siRNA and chemical screens, we assessed sensitivity of BIN67 and SCCOHT-1 to 11 RTKi. We selected a subset of RTKi, particularly those targeting FGFRs and PDGFRs. DDR assays were performed (Figure 2A and B) in an expanded panel of cell lines with known defects in SWI/SNF complex members including SMARCB1- and SMARCA2-deficient MRTs (G401 and G402) and SMARCA4 and SMARCA2-deficient lung cancer cell lines (A427 and H522), as well as the SWI/SNFwildtype human granulosa cell line, SVOG3e. While $\mathrm{IC}_{50}$ values were variable across cell lines and RTK inhibitors, these lines were most sensitive to the broad-spectrum RTKi PD-166285 and ponatinib. As ponatinib is an approved oncology drug for use in leukemias whereas PD-166285 is a tool compound, we followed up on ponatinib for the remainder of our studies.

To determine specificity of ponatinib for SMARCA4-mutant SCCOHT, we compared the $\mathrm{IC}_{50}$ values from DDR assays in SCCOHT cell lines (BIN67, SCCOHT-1, and COV434) to SWI/SNF-wildtype ovarian cancer cell lines (OVCAR-3, OVCAR-5, and OVCAR-8) as described above for drug screen hits. SCCOHT cell lines were on average nine-fold more sensitive to ponatinib than SWI/SNF-wildtype ovarian cancer cell lines, and the most sensitive SWI/SNF-wildtype ovarian cancer cell line was two-fold less sensitive than the least sensitive SCCOHT cell line (Figure 2C). We then assessed the role of SMARCA4 loss in SCCOHT sensitivity to ponatinib by utilizing a SMARCA4-mutant SCCOHT cell line, COV434, with inducible re-expression of SMARCA4. COV434 pIND20-BRG1 clones and parental COV434 cells were treated with or without doxycycline and ponatinib sensitivity was assayed by DDR. Both inducible clones showed a 1.7 -fold increase in ponatinib $\mathrm{IC}_{50}$ following SMARCA4 induction for 48 hours (Figure 2D), demonstrating that restoration of SMARCA4 induces ponatinib resistance and supporting the hypothesis that SMARCA4 loss in SCCOHT is sufficient to confer ponatinib sensitivity.

As ponatinib is known to be a broad RTKi, we sought to determine whether we could infer the ponatinib target from overlap with the siRNA screen hits. Of known ponatinib targets 
including FLT3, KIT, FGFRs, PDGFRs, RET, and ABL (32-34), only FGFR4, PDGFRA, and $P D G F R B$ were validated siRNA screen hits in BIN67 cells (Figure 2E).

\section{Known RTK targets of ponatinib are expressed in SCCOHT tumors and downstream signaling is inhibited in response to ponatinib treatment}

Because ponatinib is known to broadly target several RTKs, we sought to determine which RTKs are widely expressed in SCCOHT tumors. The expression of RTKs from RNA-Seq data on four SCCOHT primary tumors was hierarchically clustered to identify the top expressed RTKs in these cancers (Figure 3A). The top expressed genes across the tumors included two known ponatinib targets, PDGFRA and FGFR1, in addition to other RTKs such as CSF1R, EPHSA, NTRK2, and ERBB3. To functionally confirm that ponatinib inhibits signaling downstream of RTK activation, phosphorylation of p38 and Akt was examined in BIN67 cells (Figure 3B) which demonstrated a reduction in phosphorylation of p38, Akt S473 and Akt T308 following treatment with $\mathrm{IC}_{50}$ ponatinib (Figure 3B).

\section{Functional characterization of ponatinib action reveals broad kinase targeting in SCCOHT cells}

Since ponatinib is a broad RTKi, several orthogonal approaches were undertaken to identify kinases inhibited by ponatinib in SCCOHT cells. First, RTK profiler arrays were employed to screen for top RTKs inhibited by ponatinib treatment in BIN67 and SCCOHT-1. This assay is a membrane-based sandwich immunoassay that captures key RTKs and detects differential pan-tyrosine phosphorylation in cell lysates across conditions. The quantitation of these arrays is shown in Figure 4A. Of the known ponatinib targets (highlighted in yellow), PDGFRa phosphorylation was both detected at a high level relative to other RTKs and also strongly inhibited by ponatinib in BIN67 cells only, but not in SCCOHT-1 cells. This discordance between BIN67 and SCCOHT-1 would suggest that ponatinib inhibits unidentified kinases in SCCOHT cells to affect cell growth. Among previously unreported targets of ponatinib, phosphorylation of EGFR in BIN67 and SCCOHT-1 cells was greatly inhibited by ponatinib, which represented the only common candidate between the two cell lines. Other RTKs strongly inhibited by ponatinib treatment in BIN67 cells alone include RYK, CSF1R, ALK, and TEK (Figure 4A).

Given the divergent results of the RTK array assay between ponatinib sensitivity in SCCOHT-1 and BIN67, two additional proteomic approaches (MIB-MS and ABPP) were undertaken to more fully characterize functional inhibition of kinases by ponatinib. Multiplexed kinase inhibitor bead-mass spectrometry (MIB-MS) uses beads to pull down kinases from a cell lysate in the presence or absence of an inhibitor such as ponatinib $(35,36)$. Binding of ponatinib to the kinase inhibits kinase binding to MIBs. Therefore, peptides corresponding to kinase targets are lost in the subsequent mass spectrometry analyses and thereby considered hits. Due to ponatinib hypersensitivity in SCCOHT-1 cells, this line was tested using MIB-MS to identify additional candidates for ponatinib targeting (full data in Supplemental Table 4). Kinase hits from most of the major kinase families were identified in SCCOHT-1 by this approach (Figure 4B). Kinases were considered targets in this experiment if, in the vehicle control, they were over-expressed (19 kinases) or uniquely detected (24 kinases) (Figure 4B). FGFR1, FGFR2, and Src are known ponatinib targets and 
were among the differentially expressed genes. These kinases similarly displayed reduced phosphorylation in the RTK array, but only at a high dose comparable to that used for the MIB-MS analysis, suggesting that other kinases might be more strongly inhibited by ponatinib at the relevant dose for cell death. Of the RTKs highly expressed in SCCOHT tumors (Figure 3A), EphA5 was also identified as a target of ponatinib in the MIB-MS experiment (Figure 4B).

A complementary approach to determination of those kinase upon which ponatinib operates in SCCOHT, Activity-Based Protein Profiling (ABPP), uses chemical probes that covalently bind to the ATP-binding pocket of kinases and therefore can tag kinases with accessible and conformationally active ATP-binding pocket in a protein lysate prior to mass spectrometry (full data in Supplemental Tables 5 and 6) (37). The same cutoff parameters described above for MIB-MS were used for this dataset to determine unique and differentially expressed active kinases. In SCCOHT- 1 cells, we identified 13 differentially expressed kinases and 4 unique kinases in the vehicle control treated cells (Figure 4C). Only seven differentially expressed kinases in BIN67 cells were identified (Figure 4C). Two overlapping kinases were identified in both BIN67 and SCCOHT-1 cells: tyrosine kinase EphA7 and STE-family kinase LOK (Figure 4C). Interestingly, no RTK was significantly altered with ponatinib treatment in either cell line, which might represent a bias of this assay due to exclusion of active-site peptides via complementarity to other RTKs. Generally, tyrosine kinases and STE kinases were well-represented in both cell lines. All kinase hits from phospho-RTK array, MIB-MS, and APBB approaches across cell lines and PDX models tested, as well as RNA expression levels in SCCOHT tumors are summarized in Supplemental Table 7, which reiterates the conclusion of no common kinases being targets of ponatinib across cell lines.

\section{Ponatinib is effective in animal models of SCCOHT}

To determine ponatinib's promise as a potential therapy, we tested its efficacy in animal xenograft models of SCCOHT. Mice bearing subcutaneous SCCOHT-1 xenograft tumors demonstrated an initial inhibition of tumor growth following ponatinib treatment, delaying tumor progression (Figure 5A). Tumor doubling times from 200 to $400 \mathrm{~mm}^{3}$ in each group were $1.88 \pm 0.64$ days in vehicle treated mice and $7.38 \pm 1.06$ days in ponatinib treated mice (p-value < 0.0001). Treatment of SCCOHT-1 tumor bearing mice with ponatinib similarly improved median survival by $44 \%$ (Figure 5B). To further assess the efficacy of ponatinib in patient-derived tumor models, we used two SCCOHT patient-derived xenograft (PDX) models: PDX-465 and PDX-040. After 30 days of treatment, tumor growth rate was slowed in both PDX models, with statistically significant $58.6 \%$ and $42.5 \%$ decreases in tumor size relative to vehicle treatment in PDX-465 and -040, respectively (Figures 5C and 5D). To determine the pharmacodynamic effects of ponatinib in these in vivo models, RTK phosphorylation following ponatinib treatment for six hours and at the experimental endpoint ( $>30$ days) was determined (Figure 5E). Similarly to findings in SCCOHT cell lines, RTK phosphorylation was broadly reduced in each PDX model. Some RTKs overlapped PDX models (i.e. IGF1R and EGFR) and SCCOHT cell lines (i.e. EGFR and EPHA5), while others were unique to each PDX. 


\section{Discussion}

High recurrence rates, extreme toxicity, and poor overall survival in SCCOHT patients treated with standard high-dose chemotherapy and radiation necessitate the identification of effective targeted therapies for this disease. Discovery of universal disruption of SWI/SNF complex function in SCCOHT through the loss of ATPase subunits SMARCA4 and SMARCA2 has made identification of targeted therapeutic vulnerabilities possible. Further, several novel approaches to selective killing of SWI/SNF-mutant cancers have recently been explored. The SWI/SNF complex normally functions to catalyze the movement and ejection of histones to regulate the accessibility of chromatin to gene expression machinery. Antagonism between SWI/SNF and the Polycomb Repressive Complex 2 (PRC2) described in Drosophila (38), has been recapitulated in human cells where it has been specifically shown that loss of SWI/SNF function leads to a dependence on the repressive functions of PRC2 (39). For this reason, inhibitors of the PRC2 catalytic subunit, EZH2, have been examined in SMARCB1-mutant rhabdoid tumors, ARID1A-mutant ovarian clear cell carcinomas, SMARCA4-mutant non-small cell lung cancers, and SMARCA4-mutant SCCOHTs $(24,25,39-42)$. Such agents are now being examined in Phase I and II clinical trials in SMARCB1-negative or rhabdoid-like tumors, such as MRTs and SCCOHTs, but have not yet been approved for these indications. The SWI/SNF complex itself has also been identified as the major target of bromodomain inhibitors through direct interaction with bromodomain-containing SWI/SNF ATPases SMARCA2 and SMARCA4 (43). This is a particularly attractive target in some SMARCA4-deficient cancers in which residual SMARCA2 compensates in the absence of SMARCA4 as the functional SWI/SNF ATPase (44). Such an approach has been shown to be ineffective in SCCOHT, however, where expression of both SWI/SNF ATPases has been lost (3).

The effects of PRC2 dependence on tumor suppressor silencing in SWI/SNF-mutant cancers are well-studied. However, little is yet known about the effects of SWI/SNF dysfunction on the overexpression of oncogenes such as RTKs, which might cause a tumor to develop unique and targetable dependencies. Here, we show data supporting that targeting RTK activation in tumors driven by epigenetic defects may provide clinical benefit. Using HT siRNA and drug screens, our work has charted novel therapeutic vulnerabilities in SCCOHT cells. These studies have not only confirmed in vitro sensitivity to cytotoxic drug classes already utilized in SCCOHT treatment (e.g. tubulin inhibitors and DNA damaging agents), but they have also pinpointed novel drug classes, such as cardiac glycosides, not previously implicated in treatment of SWI/SNF-mutant cancers. Overall, these data converge on identification of hypersensitivity to the FDA-approved multi-kinase inhibitor ponatinib and thereby emphasize the importance of RTK signaling in these epigenetically dysregulated tumors.

We generally observed a striking similarity in drug sensitivity across the SCCOHT cell lines and SVOG3e. While this observation strengthens the case for the sensitivity of SCCOHT tumors to these agents, the similar sensitivity of SWI/SNF-wildtype SVOG3e cells indicates that most of these agents do not selectively target SWI/SNF-mutant cells. The cell of origin of SCCOHT remains unclear, but current understanding suggests derivation from a germ cell lineage (45) distinct from the cells from which SVOG3e cells were derived. Further work 
must expand cell models for each agent to better understand the relationship between cell lineage and drug response. Interestingly, previous reports have shown expression of PDGFRs and c-kit, known ponatinib targets, in granulosa cell tumors (46), suggesting convergent signaling dependencies that may account for sensitivity to this drug in SCCOHT and SVOG3e cell lines. Additional work is required to more completely characterize vulnerability of cancers with SWI/SNF loss to the agents identified in this screen.

In examining overlap between the 42 hits in the drug screen and the 109 hits in the siRNA screen, RTKs were the primary point of intersection. Network analysis of the siRNA screen data demonstrated that top enriched pathways in the screen hits were FGFR and PDGFR signaling pathways. While this screen did not include a comparator line such as a SWI/SNFwildtype ovarian cancer cell line, this comparative approach would have had a high likelihood of eliminating real therapeutic targets. RTKs and downstream kinase signaling is frequently altered in cancer, and thus finding an appropriate comparator lacking kinase dependence through a separate mechanism would be challenging to determine ahead of the screen. The role of the loss of SMARCA4 that is a defining feature of SCCOHTs for RTK dependence was demonstrated by the reduction in sensitivity of a SMARCA4-inducible SCCOHT cell line, COV434, to ponatinib.

In addition to the overlap seen within our HT screens, previous work has demonstrated sensitivity of SWI/SNF-mutant MRTs to ponatinib (30). Mechanistically, our work supports data reported in SMARCB1-mutant MRTs, in which loss of the core SWI/SNF subunit SMARCB1 results in elevated FGFR or PDGFR expression (26,30). We similarly observed sensitivity of SCCOHT cell lines to ponatinib, a broad RTKi, as well as other RTK inhibitors with more specific drug profiles, such as PD-166285. PD-166285, a Pfizer compound developed twenty years ago (47), has shown limited promise in preclinical animal models (48) and in vitro efficacy in other cancer models (49), but has not been further developed towards clinical use. Despite PD-166285 being the most effective RTKi tested, the promise of this agent towards clinical development in a rare cancer precludes its clinical utility, and was therefore not further studied in the context of a SCCOHT therapeutic agent. Ponatinib was further prioritized based on existing oncology approval for clinical use in chronic myeloid leukemia and acute lymphoblastic leukemia.

Multiple methods were integrated in an attempt to functionally identify a common target of ponatinib responsible for efficacy of this drug in SCCOHT. Criteria considered included: 1) expression in SCCOHT primary tumors, 2) requirement of expression for cell growth, and 3) functional inhibition in the presence of ponatinib. Using these criteria, however, no common target was identified, both between the extensive methods described here and the two SCCOHT cell lines. Furthermore, the sensitivity of SCCOHT cells to other RTKi is inversely related to the specificity of the RTKi to its intended target. Together, this evidence suggests general reliance of SCCOHT on multiple kinases such that a broad kinase inhibitor such as ponatinib is effective.

Similar findings of broad RTK inhibition were found in the pharmacodynamics analysis of the two PDX models treated with ponatinib. Within six hours of treatment in vivo, seventeen and five RTKs were inhibited by greater than two-fold in PDX-040 and PDX-465, 
respectively. The phosphorylation of many additional RTKs were reduced to intermediate levels as well. Following > 30 days of treatment with ponatinib, tumor growth was significantly inhibited, but was not completely blocked. This might be partially explained by lack of a sustained inhibition of RTK signaling, as 3/17 (18\%) and 0/5 (0\%) of these initially inhibited RTKs also demonstrated reductions in phosphorylation of greater than two-fold at the efficacy endpoint. Future studies will be needed to determine if this lack of sustained RTK inhibition is the cause of incomplete tumor growth inhibition and whether drug dosing changes, such as more intermittent dosing, might improve drug response.

When taken in combination with similar findings previously reported in MRTs $(26,27,30)$ and the observations in other SWI/SNF mutant lung cancer cell lines reported here, we believe findings on ponatinib in SCCOHT and MRT models might be more broadly applicable to a larger set of "SWI/SNF-omas", or cancers with mutations in the SWI/SNF complex. This group accounts for up to $20 \%$ of all cancers, and therefore has potential for broader translational relevance. Our work also demonstrates the efficacy of ponatinib in SCCOHT preclinical animal models. Ponatinib was granted accelerated approval by the FDA in 2012 for use in leukemias, but was briefly taken off the market after observation of serious vascular events. The FDA granted full approval at the end of 2016 after completion of the Phase II PACE clinical trial. Ponatinib is currently being assessed for efficacy in a number of other cancers, including solid tumors, in ongoing clinical trials. This work demonstrates significant promise for ponatinib in improving SCCOHT patient outcomes and warrants clinical investigation.

\section{Supplementary Material}

Refer to Web version on PubMed Central for supplementary material.

\section{Acknowledgements}

This work is supported by the National Institutes of Health (1R01CA195670-01, to D.G.H., J.T. and B.W.), the Canadian Cancer Society Research Institute (\#703458, to D.G.H.), the Terry Fox Research Institute Initiative New Frontiers Program in Cancer (D.G.H.), the Marsha Rivkin Center for Ovarian Cancer Research, the Ovarian Cancer Alliance of Arizona, the Small Cell Ovarian Cancer Foundation, and philanthropic support to the TGen Foundation. Thank you to Drs. Michael Humphries and Joseph Gozgit at ARIAD Pharmaceuticals for their thoughtful discussions on ponatinib. We would also like to thank the SCCOHT patients, their families and communities, and the clinicians who have contributed significantly to the motivation and feasibility of this work.

Grant Support

This work was supported by research funds from the Canadian Cancer Society Research Institute (\#703458, D.G.H.), the National Institutes of Health (R01 CA195670-01, B.E.W., D.G.H., and J.M.T., and T32 HL007106-39 to E.M.C), the Terry Fox Research Institute Initiative New Frontiers Program in Cancer (\#1021, D.G.H.), the British Columbia Cancer Foundation (D.G.H.), the VGH \& UBC Foundation (D.G.H.), the Anne Rita Monahan Foundation (P.R.), the Marsha Rivkin Center for Ovarian Cancer Research (J.M.T.), the Ovarian Cancer Alliance of Arizona (J.M.T.), the Small Cell Ovarian Cancer Foundation (P.R., J.D.L., B.V., and J.M.T.), and philanthropic support to the TGen Foundation (J.M.T.).

\section{References}

1. Witkowski L, Goudie C, Ramos P, Boshari T, Brunet JS, Karnezis AN, et al. The influence of clinical and genetic factors on patient outcome in small cell carcinoma of the ovary, hypercalcemic type. Gynecol Oncol 2016;141(3):454-60 doi 10.1016/j.ygyno.2016.03.013. [PubMed: 26975901] 
2. Estel R, Hackethal A, Kalder M, Munstedt K. Small cell carcinoma of the ovary of the hypercalcaemic type: an analysis of clinical and prognostic aspects of a rare disease on the basis of cases published in the literature. Arch Gynecol Obstet 2011;284(5):1277-82 doi 10.1007/ s00404-011-1846-5. [PubMed: 21298438]

3. Karnezis AN, Wang Y, Ramos P, Hendricks WP, Oliva E, D'Angelo E, et al. Dual loss of the SWI/SNF complex ATPases SMARCA4/BRG1 and SMARCA2/BRM is highly sensitive and specific for small cell carcinoma of the ovary, hypercalcaemic type. The Journal of pathology 2016;238(3):389-400. [PubMed: 26356327]

4. Ramos P, Karnezis AN, Craig DW, Sekulic A, Russell ML, Hendricks WP, et al. Small cell carcinoma of the ovary, hypercalcemic type, displays frequent inactivating germline and somatic mutations in SMARCA4. Nature genetics 2014;46(5):427-9 doi 10.1038/ng.2928. [PubMed: 24658001]

5. Ramos P, Karnezis AN, Hendricks WP, Wang Y, Tembe W, Zismann VL, et al. Loss of the tumor suppressor SMARCA4 in small cell carcinoma of the ovary, hypercalcemic type (SCCOHT). Rare Diseases 2014;2(1):e967148. [PubMed: 26942101]

6. Jelinic P, Mueller JJ, Olvera N, Dao F, Scott SN, Shah R, et al. Recurrent SMARCA4 mutations in small cell carcinoma of the ovary. Nature genetics 2014;46(5):424-6 doi 10.1038/ng.2922. [PubMed: 24658004]

7. Jelinic P, Schlappe BA, Conlon N, Tseng J, Olvera N, Dao F, et al. Concomitant loss of SMARCA2 and SMARCA4 expression in small cell carcinoma of the ovary, hypercalcemic type. Modern Pathology 2015;29(1):60-6. [PubMed: 26564006]

8. Witkowski L, Carrot-Zhang J, Albrecht S, Fahiminiya S, Hamel N, Tomiak E, et al. Germline and somatic SMARCA4 mutations characterize small cell carcinoma of the ovary, hypercalcemic type. Nature genetics 2014;46(5):438-43 doi 10.1038/ng.2931. [PubMed: 24658002]

9. Le Loarer F, Watson S, Pierron G, de Montpreville VT, Ballet S, Firmin N, et al. SMARCA4 inactivation defines a group of undifferentiated thoracic malignancies transcriptionally related to BAF-deficient sarcomas. Nat Genet 2015;47(10):1200-5 doi 10.1038/ng.3399. [PubMed: 26343384]

10. Biegel JA, Zhou JY, Rorke LB, Stenstrom C, Wainwright LM, Fogelgren B. Germ-line and acquired mutations of INI1 in atypical teratoid and rhabdoid tumors. Cancer Res 1999;59(1):74-9. [PubMed: 9892189]

11. Kahali B, Yu J, Marquez SB, Thompson KW, Liang SY, Lu L, et al. The silencing of the SWI/SNF subunit and anticancer gene BRM in Rhabdoid tumors. Oncotarget 2014;5(10):3316-32 doi 10.18632/oncotarget.1945. [PubMed: 24913006]

12. Cheng JX, Tretiakova M, Gong C, Mandal S, Krausz T, Taxy JB. Renal medullary carcinoma: rhabdoid features and the absence of INI1 expression as markers of aggressive behavior. Mod Pathol 2008;21(6):647-52 doi 10.1038/modpathol.2008.44. [PubMed: 18327209]

13. Calderaro J, Masliah-Planchon J, Richer W, Maillot L, Maille P, Mansuy L, et al. Balanced Translocations Disrupting SMARCB1 Are Hallmark Recurrent Genetic Alterations in Renal Medullary Carcinomas. Eur Urol 2016;69(6):1055-61 doi 10.1016/j.eururo.2015.09.027. [PubMed: 26433572]

14. Jones S, Wang TL, Shih Ie M, Mao TL, Nakayama K, Roden R, et al. Frequent mutations of chromatin remodeling gene ARID1A in ovarian clear cell carcinoma. Science 2010;330(6001): 228-31 doi 10.1126/science.1196333. [PubMed: 20826764]

15. Wiegand KC, Shah SP, Al-Agha OM, Zhao Y, Tse K, Zeng T, et al. ARID1A mutations in endometriosis-associated ovarian carcinomas. N Engl J Med 2010;363(16):1532-43 doi 10.1056/ NEJMoa1008433. [PubMed: 20942669]

16. Reisman DN, Sciarrotta J, Wang W, Funkhouser WK, Weissman BE. Loss of BRG1/BRM in human lung cancer cell lines and primary lung cancers: correlation with poor prognosis. Cancer research 2003;63(3):560-6. [PubMed: 12566296]

17. Orvis T, Hepperla A, Walter V, Song S, Simon J, Parker J, et al. BRG1/SMARCA4 inactivation promotes non-small cell lung cancer aggressiveness by altering chromatin organization. Cancer Res 2014;74(22):6486-98 doi 10.1158/0008-5472.CAN-14-0061. [PubMed: 25115300] 
18. Shain AH, Pollack JR. The spectrum of SWI/SNF mutations, ubiquitous in human cancers. PloS one 2013;8(1):e55119 doi 10.1371/journal.pone.0055119. [PubMed: 23355908]

19. Kadoch C, Hargreaves DC, Hodges C, Elias L, Ho L, Ranish J, et al. Proteomic and bioinformatic analysis of mammalian SWI/SNF complexes identifies extensive roles in human malignancy. Nature genetics 2013;45(6):592-601 doi 10.1038/ng.2628. [PubMed: 23644491]

20. Otte A, Rauprich F, Hillemanns P, Park-Simon T-W, von der Ohe J, Hass R. In vitro and in vivo therapeutic approach for a small cell carcinoma of the ovary hypercalcaemic type using a SCCOHT-1 cellular model. Orphanet journal of rare diseases 2014;9(1):1. [PubMed: 24393603]

21. Otte A, Rauprich F, von der Ohe J, Yang Y, Kommoss F, Feuerhake F, et al. c-Met inhibitors attenuate tumor growth of small cell hypercalcemic ovarian carcinoma (SCCOHT) populations. Oncotarget 2015;6(31):31640-58 doi 10.18632/oncotarget.5151. [PubMed: 26436697]

22. Gamwell LF, Gambaro K, Merziotis M, Crane C, Arcand SL, Bourada V, et al. Small cell ovarian carcinoma: genomic stability and responsiveness to therapeutics. Orphanet J Rare Dis 2013;8:33. [PubMed: 23433318]

23. Filippakopoulos P, Knapp S. Targeting bromodomains: epigenetic readers of lysine acetylation. Nat Rev Drug Discov 2014;13(5):337-56 doi 10.1038/nrd4286. [PubMed: 24751816]

24. Chan-Penebre E, Armstrong K, Drew A, Grassian AR, Feldman I, Knutson SK, et al. Selective Killing of SMARCA2- and SMARCA4-deficient Small Cell Carcinoma of the Ovary, Hypercalcemic Type Cells by Inhibition of EZH2: In Vitro and In Vivo Preclinical Models. Mol Cancer Ther 2017;16(5):850-60 doi 10.1158/1535-7163.MCT-16-0678. [PubMed: 28292935]

25. Wang Y, Chen SY, Karnezis AN, Colborne S, Santos ND, Lang JD, et al. The histone methyltransferase EZH2 is a therapeutic target in small cell carcinoma of the ovary, hypercalcemic type. J Pathol 2017 doi 10.1002/path.4912.

26. Wohrle S, Weiss A, Ito M, Kauffmann A, Murakami M, Jagani Z, et al. Fibroblast growth factor receptors as novel therapeutic targets in SNF5-deleted malignant rhabdoid tumors. PLoS One 2013;8(10):e77652 doi 10.1371/journal.pone.0077652. [PubMed: 24204904]

27. Isakoff MS, Sansam CG, Tamayo P, Subramanian A, Evans JA, Fillmore CM, et al. Inactivation of the Snf5 tumor suppressor stimulates cell cycle progression and cooperates with p53 loss in oncogenic transformation. Proc Natl Acad Sci U S A 2005;102(49):17745-50 doi 10.1073/pnas. 0509014102. [PubMed: 16301525]

28. Darr J, Klochendler A, Isaac S, Eden A. Loss of IGFBP7 expression and persistent AKT activation contribute to SMARCB1/Snf5-mediated tumorigenesis. Oncogene 2014;33(23):3024-32 doi 10.1038/onc.2013.261. [PubMed: 23851500]

29. Charboneau A, Chai J, Jordan J, Funkhouser W, Judkins A, Biegel J, et al. P-Akt expression distinguishes two types of malignant rhabdoid tumors. J Cell Physiol 2006;209(2):422-7 doi 10.1002/jcp.20737. [PubMed: 16897758]

30. Wong JP, Todd JR, Finetti MA, McCarthy F, Broncel M, Vyse S, et al. Dual Targeting of PDGFRalpha and FGFR1 Displays Synergistic Efficacy in Malignant Rhabdoid Tumors. Cell Rep 2016;17(5):1265-75 doi 10.1016/j.celrep.2016.10.005. [PubMed: 27783942]

31. Wu G, Feng X, Stein L. A human functional protein interaction network and its application to cancer data analysis. Genome Biol 2010;11(5):R53 doi 10.1186/gb-2010-11-5-r53. [PubMed: 20482850]

32. Gozgit JM, Wong MJ, Wardwell S, Tyner JW, Loriaux MM, Mohemmad QK, et al. Potent activity of ponatinib (AP24534) in models of FLT3-driven acute myeloid leukemia and other hematologic malignancies. Mol Cancer Ther 2011;10(6):1028-35 doi 10.1158/1535-7163.MCT-10-1044. [PubMed: 21482694]

33. De Falco V, Buonocore P, Muthu M, Torregrossa L, Basolo F, Billaud M, et al. Ponatinib (AP24534) is a novel potent inhibitor of oncogenic RET mutants associated with thyroid cancer. $\mathrm{J}$ Clin Endocrinol Metab 2013;98(5):E811-9 doi 10.1210/jc.2012-2672. [PubMed: 23526464]

34. Huang WS, Metcalf CA, Sundaramoorthi R, Wang Y, Zou D, Thomas RM, et al. Discovery of 3[2-(imidazo[1,2-b]pyridazin-3-yl)ethynyl]-4-methyl-N-\{4-[(4-methylpiperazin-1-y l)methyl]-3(trifluoromethyl)phenyl $\}$ benzamide (AP24534), a potent, orally active pan-inhibitor of breakpoint cluster region-abelson (BCR-ABL) kinase including the T315I gatekeeper mutant. J Med Chem 2010;53(12):4701-19 doi 10.1021/jm100395q. [PubMed: 20513156] 
35. Cooper MJ, Cox NJ, Zimmerman EI, Dewar BJ, Duncan JS, Whittle MC, et al. Application of multiplexed kinase inhibitor beads to study kinome adaptations in drug-resistant leukemia. PLoS One 2013;8(6):e66755 doi 10.1371/journal.pone.0066755. [PubMed: 23826126]

36. Duncan JS, Whittle MC, Nakamura K, Abell AN, Midland AA, Zawistowski JS, et al. Dynamic reprogramming of the kinome in response to targeted MEK inhibition in triple-negative breast cancer. Cell 2012;149(2):307-21 doi 10.1016/j.cell.2012.02.053. [PubMed: 22500798]

37. Patricelli MP, Szardenings AK, Liyanage M, Nomanbhoy TK, Wu M, Weissig H, et al. Functional interrogation of the kinome using nucleotide acyl phosphates. Biochemistry 2007;46(2):350-8 doi 10.1021/bi062142x. [PubMed: 17209545]

38. Kennison JA. The Polycomb and trithorax group proteins of Drosophila: trans-regulators of homeotic gene function. Annu Rev Genet 1995;29:289-303 doi 10.1146/annurev.ge. 29.120195.001445. [PubMed: 8825476]

39. Wilson BG, Wang X, Shen X, McKenna ES, Lemieux ME, Cho YJ, et al. Epigenetic antagonism between polycomb and SWI/SNF complexes during oncogenic transformation. Cancer Cell 2010;18(4):316-28 doi 10.1016/j.ccr.2010.09.006. [PubMed: 20951942]

40. Bitler BG, Aird KM, Garipov A, Li H, Amatangelo M, Kossenkov AV, et al. Synthetic lethality by targeting EZH2 methyltransferase activity in ARID1A-mutated cancers. Nat Med 2015;21(3):2318 doi 10.1038/nm.3799. [PubMed: 25686104]

41. Bitler BG, Aird KM, Zhang R. Epigenetic synthetic lethality in ovarian clear cell carcinoma: EZH2 and ARID1A mutations. Mol Cell Oncol 2016;3(1):e1032476 doi 10.1080/23723556.2015.1032476. [PubMed: 27308548]

42. Kim KH, Kim W, Howard TP, Vazquez F, Tsherniak A, Wu JN, et al. SWI/SNF-mutant cancers depend on catalytic and non-catalytic activity of EZH2. Nat Med 2015;21(12):1491-6 doi 10.1038/nm.3968. [PubMed: 26552009]

43. Vangamudi B, Paul TA, Shah PK, Kost-Alimova M, Nottebaum L, Shi X, et al. The SMARCA2/4 ATPase Domain Surpasses the Bromodomain as a Drug Target in SWI/SNF-Mutant Cancers: Insights from cDNA Rescue and PFI-3 Inhibitor Studies. Cancer research 2015;75(18):3865-78 doi 10.1158/0008-5472.CAN-14-3798. [PubMed: 26139243]

44. Wilson BG, Helming KC, Wang X, Kim Y, Vazquez F, Jagani Z, et al. Residual complexes containing SMARCA2 (BRM) underlie the oncogenic drive of SMARCA4 (BRG1) mutation. Molecular and cellular biology 2014 doi 10.1128/MCB.01372-13.

45. Kupryjanczyk J, Dansonka-Mieszkowska A, Moes-Sosnowska J, Plisiecka-Halasa J, Szafron L, Podgorska A, et al. Ovarian small cell carcinoma of hypercalcemic type - evidence of germline origin and SMARCA4 gene inactivation. A pilot study. Pol J Pathol 2013;64(4):238-46. [PubMed: 24375037]

46. Rocconi RP, Matthews KS, Kimball KJ, Conner MG, Baker AC, Barnes MN. Expression of c-kit and platelet-derived growth factor receptors in ovarian granulosa cell tumors. Reprod Sci 2008;15(7):673-7 doi 10.1177/1933719108317584. [PubMed: 18492696]

47. Panek RL, Lu GH, Klutchko SR, Batley BL, Dahring TK, Hamby JM, et al. In vitro pharmacological characterization of PD 166285, a new nanomolar potent and broadly active protein tyrosine kinase inhibitor. J Pharmacol Exp Ther 1997;283(3):1433-44. [PubMed: 9400019]

48. Dimitroff CJ, Klohs W, Sharma A, Pera P, Driscoll D, Veith J, et al. Anti-angiogenic activity of selected receptor tyrosine kinase inhibitors, PD166285 and PD173074: implications for combination treatment with photodynamic therapy. Invest New Drugs 1999;17(2):121-35. [PubMed: 10638483]

49. Kogiso T, Nagahara H, Hashimoto E, Ariizumi S, Yamamoto M, Shiratori K. Efficient induction of apoptosis by wee1 kinase inhibition in hepatocellular carcinoma cells. PLoS One 2014;9(6):e100495 doi 10.1371/journal.pone.0100495. [PubMed: 24960176] 


\section{Statement of Translational Relevance}

Pathogenic mutations in SWI/SNF chromatin-remodeling complex members occur in approximately $20 \%$ of cancers, but no targeted therapies that exploit a tumor's dependence on SWI/SNF dysfunction have yet shown clinical impact. Small cell carcinoma of the ovary, hypercalcemic type (SCCOHT) is a rare and aggressive form of ovarian cancer affecting young women. It is characterized by mutational inactivation of SMARCA4 and epigenetic silencing of SMARCA2, the mutually exclusive ATPases of the SWI/SNF complex. Here, we demonstrate potency of the FDA-approved oncology drug, ponatinib, a receptor tyrosine kinase (RTK) inhibitor whose targets include PDGFRs, FGFRs, and EphAs, in SMARCA4-mutant SCCOHT cell and animal models. This work suggests that ponatinib exploits SCCOHT's dependence on RTK signaling in the context of SWI/SNF dysregulation and that ponatinib may be effective in SCCOHT treatment. Preclinical identification of an effective, approved oncology drug holds promise for rapidly improving outcomes for these young patients and warrants clinical investigation. 


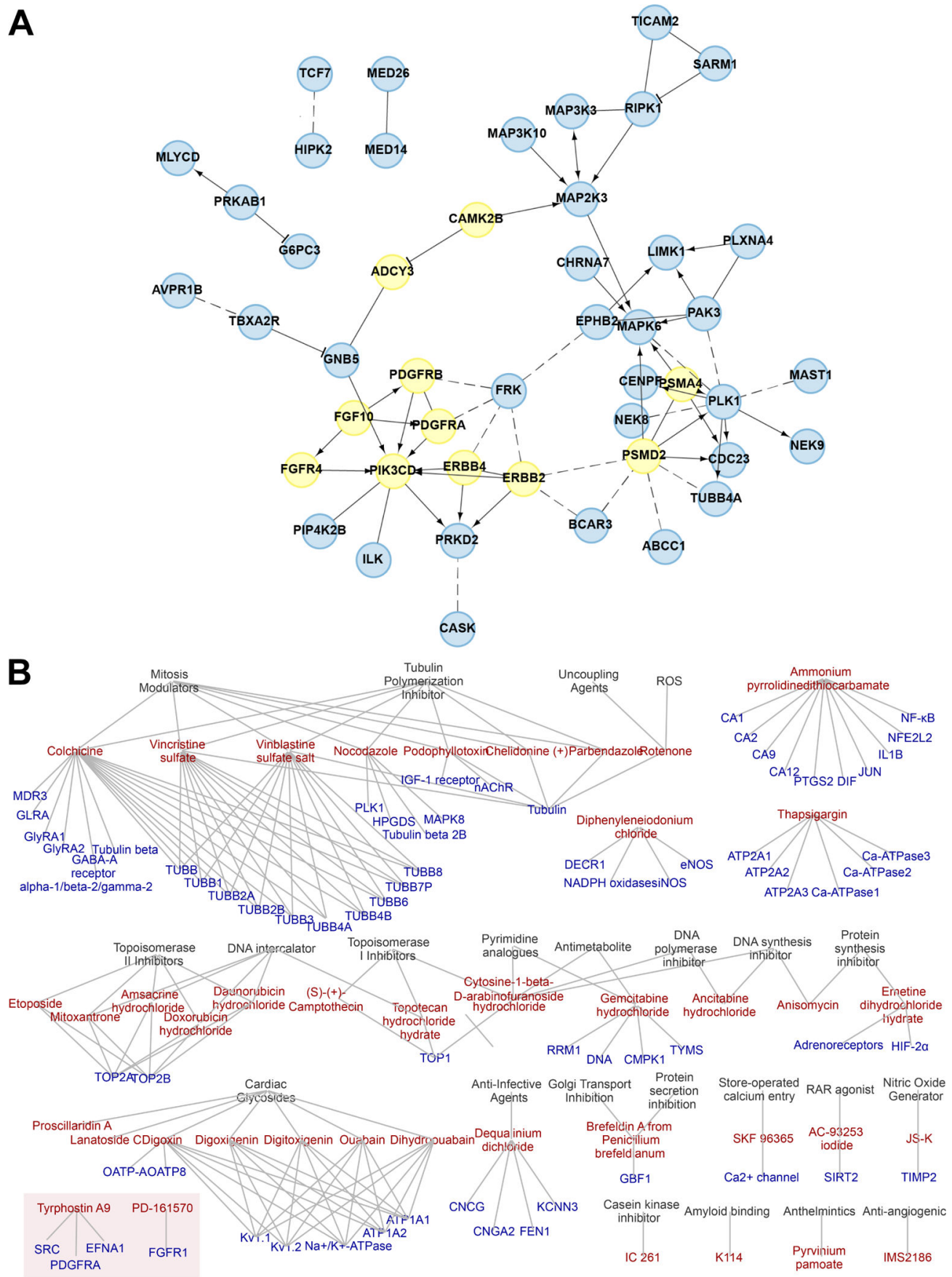

Figure 1. High-throughput screen network analysis.

(A) Protein interaction network constructed for RNAi validated screen hits using ReactomeFI network resources, as further described in the supplemental materials and methods. Curated interactions are represented by solid edges with arrows for interactions involved in catalysis or activation and edges with " $\mathrm{T}$ ” bar for inhibitory connections. Dotted edges are those interactions that are computationally predicted. Nodes in the network represent validate RNAi hits. Nodes with no edges are not pictured here (full validated hit list in Supplemental Table 3). Genes that were identified as PDGFR pathway members by 
ReactomeFI are highlighted in yellow, all other genes colored in blue. (B) Chemical hit entity association network for verified chemical hits and their targets and associated biological concepts in SCCOHT cell line, BIN67. RTK associated hits are highlighted in box. 

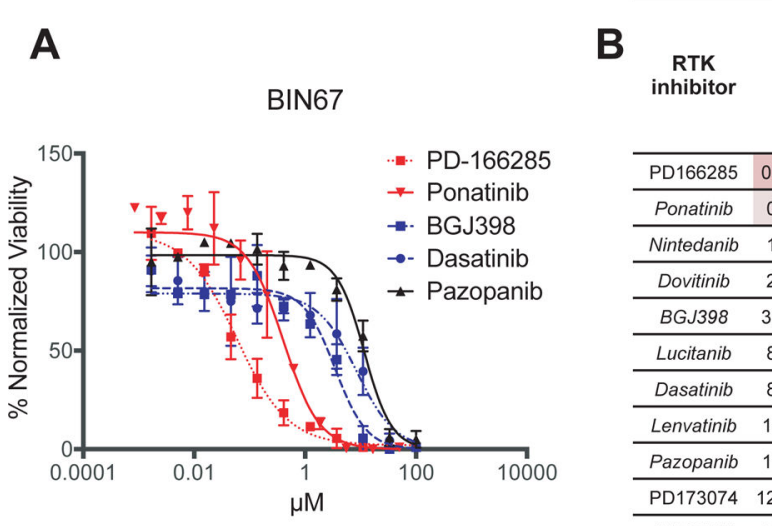

\begin{tabular}{|c|c|c|c|c|c|c|c|}
\hline \multirow[b]{2}{*}{$\begin{array}{c}\text { RTK } \\
\text { inhibitor }\end{array}$} & \multicolumn{7}{|c|}{$\mathrm{IC}_{50}(\boldsymbol{\mu} \mathrm{M})$} \\
\hline & $\frac{\hat{o}}{\underline{m}}$ & 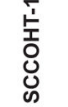 & 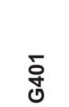 & ণ̃ & 产 & $\begin{array}{l}\text { ิㅗ } \\
\text { ํ. }\end{array}$ & $\begin{array}{l}\stackrel{0}{0} \\
\text { ఏ } \\
\text { गे }\end{array}$ \\
\hline PD166285 & 0.0648 & 0.004 & 0.094 & 0.033 & 0.097 & 0.251 & 0.010 \\
\hline Ponatinib & 0.333 & 0.005 & 0.233 & 0.046 & 0.098 & 0.457 & 0.108 \\
\hline Nintedanib & 1.365 & 0.128 & & & & & 1.027 \\
\hline Dovitinib & 2.203 & 0.035 & & & & & 0.404 \\
\hline BGJ398 & 3.6765 & 0.002 & 2.0405 & 0.011 & 0.003 & 3.553 & 0.441 \\
\hline Lucitanib & 8.395 & 0.015 & & & & & \\
\hline Dasatinib & 8.650 & 1.300 & & & & & 0.025 \\
\hline Lenvatinib & 11.542 & 0.069 & & & & & 5.110 \\
\hline Pazopanib & 11.690 & 0.110 & & & & & 3.953 \\
\hline PD173074 & 12.3005 & 0.0005 & 9.271 & 0.247 & 0.006 & 7.071 & \\
\hline AZD4547 & 14.540 & 0.002 & & & & & 0.876 \\
\hline
\end{tabular}
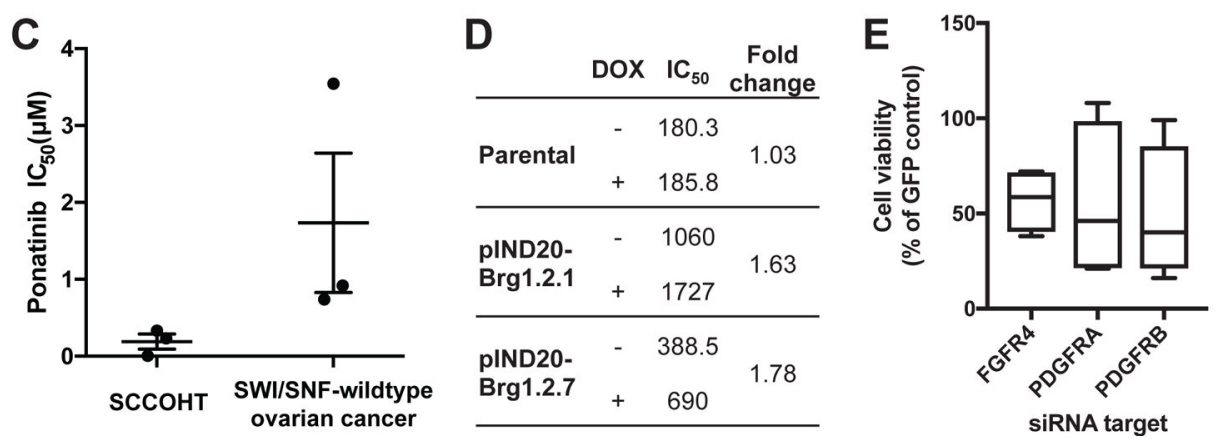

Figure 2. Sensitivity of SCCOHT and other SWI/SNF mutant cell lines to RTK inhibition. DDR curves for various RTKi in BIN67 cells. Cell viability was assayed after 72 hours treatment. (B) Summary of $\mathrm{IC}_{50}$ values for RTKi tested in SCCOHT cell lines, along with selected agents tested in SWI/SNF-mutant MRT cell lines (G401 and G402) and lung cancer cell lines (A427 and H522) vs SWI/SNF-wildtype immortalized granulosa cells (SVOG3e). Conditions not tested are in gray. (C) Comparison of $\mathrm{IC}_{50}$ values for ponatinib in SCCOHT cell lines (BIN67, SCCOHT-1, and COV434) versus ovarian cancer cell lines selected for their wildtype SWI/SNF status (OVCAR-3, OVCAR-5, and OVCAR-8). (D) Fold change in ponatinib $\mathrm{IC}_{50}$ value following doxycycline treatment in parental COV434 cells and two COV434 pIND20 BRG1 clones (inducible SMARCA4). (E) Cell viability of BIN67 cells following knockdown of ponatinib target genes. Known ponatinib target genes that were initial hits in the siRNA screen (FGFR4, PDGFRA, and PDGFRB) were validated in a secondary siRNA screen. Data shown is mean cell viability of 3 independent replicates of 4 distinct siRNAs relative to internal GFP-targeting siRNA control. All three target genes were considered validated hits based on criteria described in methods. 


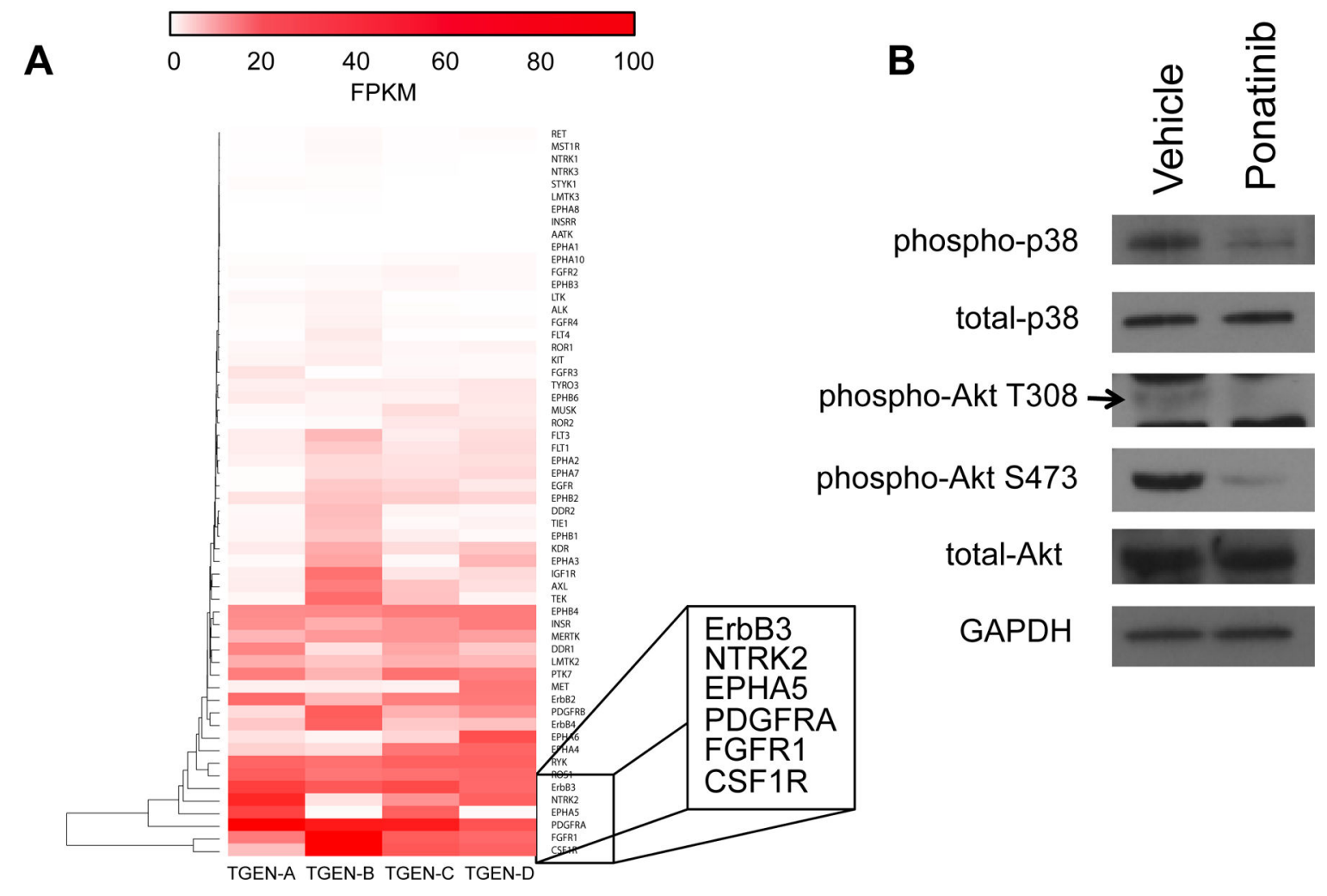

Figure 3. RTK expression in SCCOHT primary tumors and downstream RTK signaling inhibited by ponatinib in SCCOHT cells.

(A) Expression of all RTK genes in four SCCOHT primary tumors from RNA-Seq. Expression is displayed in FPKM, and genes are hierarchically clustered. The six most highly expressed RTKs are in inset. (B) Phosphorylation of signaling components downstream of RTKs are inhibited by ponatinib within 30 min treatment in BIN67 cells. 
A

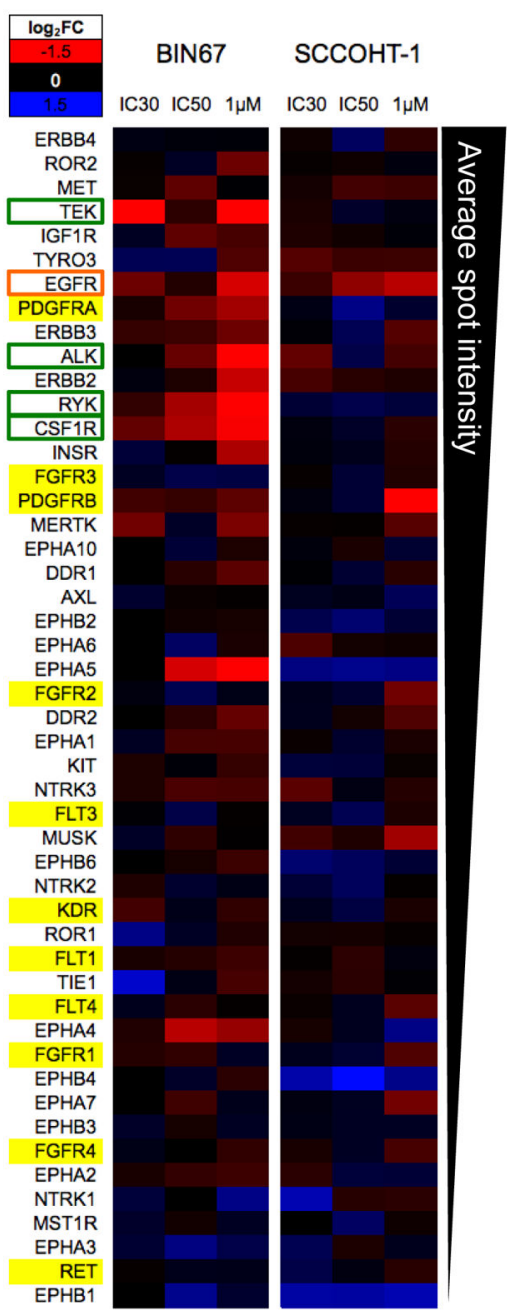

B
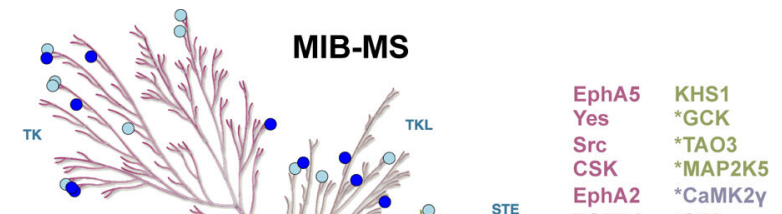

EphA2 *CaMK2

FGFR1 *SIK

FGFR2 p38 $\beta$

*EphB3 PCTAIRE2

*EphA3 "p38a

*FYN ${ }^{*}$ CLK1

${ }^{*} \mathrm{ABL1} \quad \mathrm{IKKa}$

${ }^{*} \mathrm{ABL2} \quad \mathrm{IKK} \beta$

*ABL2

*JAK3

IRAK

LIMK1

BRAF

*RIPK2

*ZAK

*TAK1

*TGF $\beta R 2$

MEKK3

HGKIZC1

LOK

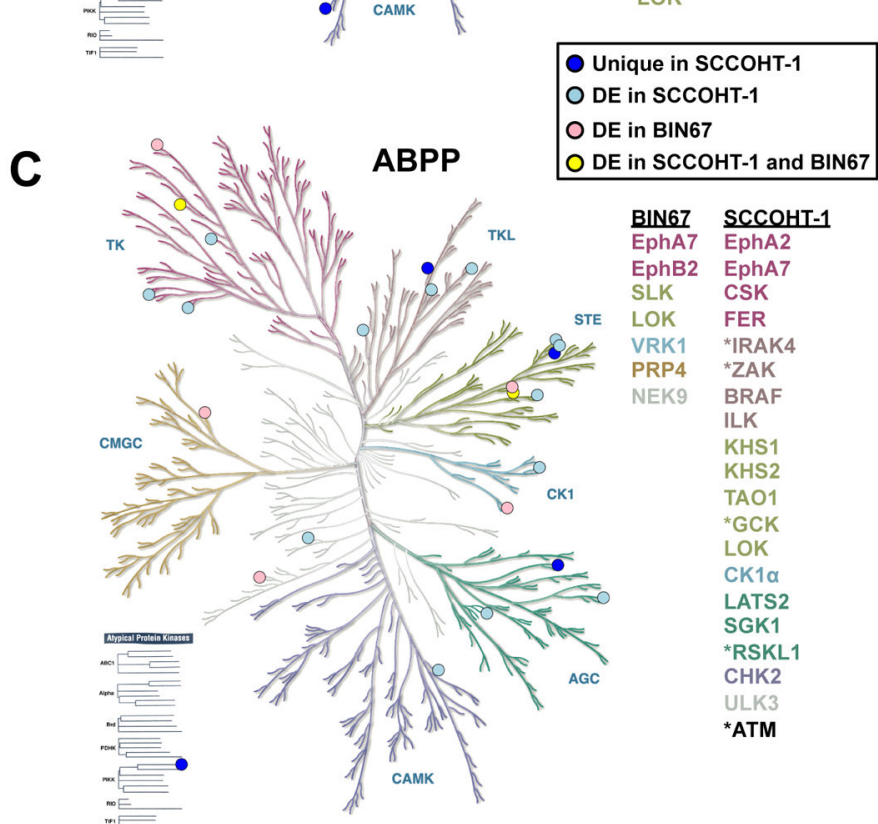

Figure 4. RTK signaling inhibited by ponatinib in SCCOHT cells.

(A) Phospho-RTK array performed on lysates from BIN67 cells treated with ponatinib for 1 hour. Background-normalized spot intensity relative to vehicle-treated control is represented as a heatmap, where blue indicates an increase in phosphorylation and red indicates a decrease. RTKs are arranged from top to bottom based on mean basal dot intensity. Known ponatinib targets are highlighted in yellow, kinases inhibited in both cell lines are outlined in orange and kinases inhibited in one cell line only are outlined in green. (B and $\mathrm{C}$ ) Kinases identified from MIB-MS assay (B) and (C) ABPP assay in SCCOHT-1 cells (blue circles, $2 \mu \mathrm{M}$, biological triplicates) and BIN67 cells (red circles, $\mathrm{IC}_{50}$, technical replicates) or in both cell lines (yellow circles) mapped to kinase dendrogram. The gene lists are color-coded based on corresponding kinase family in the dendrogram. Atypical protein kinases are listed in black. Differentially expressed kinases are shown in lighter shaded circles. Uniquely expressed kinases are shown in darker shaded circles and are marked with an asterisk in the gene list. 
A

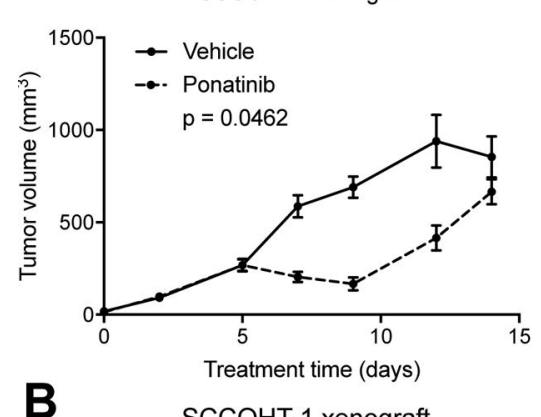

B

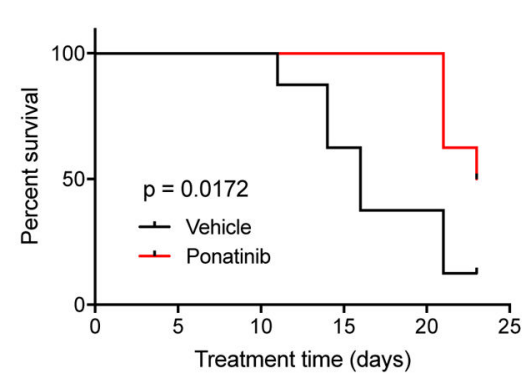

C
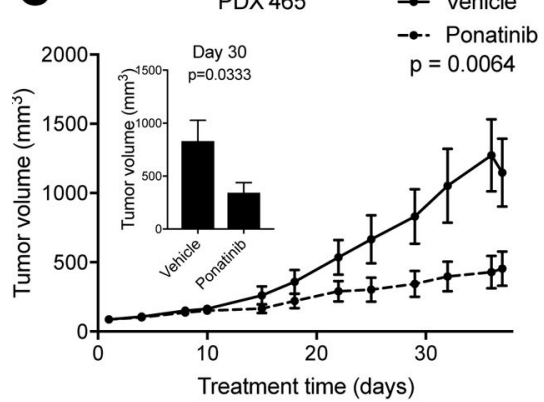

D

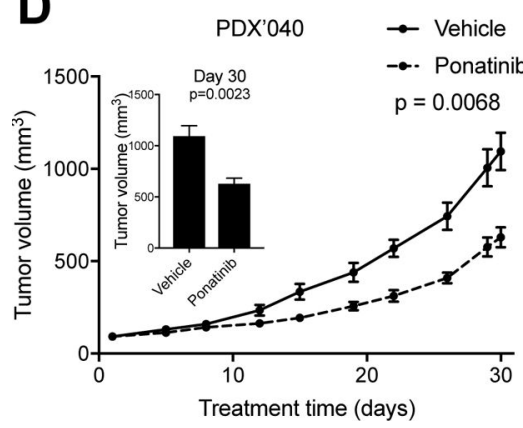

E

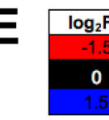

$\log _{2} \mathrm{FC}$
-1.5
0
1.5

PDX'040

PDX'465

EPHA10

TYRO3

ROR2

INSR

EPHA1

IGF1R

CSF1R

KDR

ROR1

EPHA5

DDR2

DDR1

EGFR

MERTK

MUSK

FLT4

EPHB3

EPHB6

KIT

PDGFRB

FGFR3

EPHB4

MST1R

RYK

TEK

RET

FGFR2

ERBB4

ERBB3

NTRK2

FLT1

EPHB2

FGFR1

FGFR4

FLT3

ALK

EPHB1

EPHA2

EPHA6

MET

AXL

EPHA7

TIE1

EPHA4

NTRK3

NTRK1

PDGFRA

ERBB2

EPHA3
6 hour Endpoint
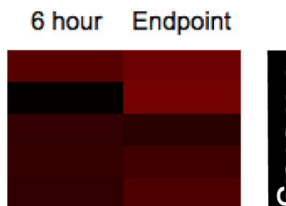

永

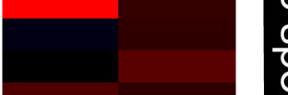

Figure 5. Efficacy of ponatinib in animal models of SCCOHT.

(A) SCCOHT-1 xenograft growth with treatment with vehicle or ponatinib. (B) Survival of SCCOHT-1 xenograft-bearing mice following treatment with vehicle or ponatinib. (C and D) Tumor volumes of SCCOHT PDX model '465 (C) or '040 (D) growth with treatment with vehicle or ponatinib. Insets in (C) and (D) show tumor volumes at day 30. (E) Phospho-RTK array performed on lysates from flash-frozen PDX tumor tissues following treatment with 
ponatinib for six hours or at the experimental endpoint for the efficacy studies in Figure 5C and 5D and analyzed as in Figure 4A. Data represents the average of two tumors per group. 
Table 1.

Top pathways enriched in siRNA screen gene hits using ReactomeFI (Cytoscape).

\begin{tabular}{lccccc}
\hline \multicolumn{1}{c}{ Gene Set } & $\begin{array}{c}\text { Ratio of } \\
\text { Proteins in } \\
\text { Gene Set }\end{array}$ & $\begin{array}{c}\text { Number of } \\
\text { Proteins In } \\
\text { Gene Set }\end{array}$ & Proteins from Network & p-value & FDR \\
\hline PIP3 activates AKT signaling (R) & 0.0093 & 95 & 8 & $1.56 \mathrm{E}-06$ & $2.72 \mathrm{E}-04$ \\
Signaling by FGFR4 (R) & 0.0269 & 275 & 12 & $3.49 \mathrm{E}-06$ & $2.72 \mathrm{E}-04$ \\
Signaling by FGFR3 (R) & 0.0269 & 275 & 12 & $3.49 \mathrm{E}-06$ & $2.72 \mathrm{E}-04$ \\
Signaling by FGFR2 (R) & 0.027 & 276 & 12 & $3.62 \mathrm{E}-06$ & $2.72 \mathrm{E}-04$ \\
Signaling by FGFR1 (R) & 0.027 & 276 & 12 & $3.62 \mathrm{E}-06$ & $2.72 \mathrm{E}-04$ \\
Signaling by ERBB2 (R) & 0.0272 & 278 & 12 & $3.89 \mathrm{E}-06$ & $2.72 \mathrm{E}-04$ \\
Signaling by Interleukins (R) & 0.0274 & 280 & 12 & $4.19 \mathrm{E}-06$ & $2.72 \mathrm{E}-04$ \\
Signaling by EGFR (R) & 0.029 & 296 & 12 & $7.32 \mathrm{E}-06$ & $3.91 \mathrm{E}-04$ \\
DAP12 interactions (R) & 0.0292 & 298 & 12 & $7.83 \mathrm{E}-06$ & $3.91 \mathrm{E}-04$ \\
Signaling by PDGF (R) & 0.0296 & 302 & 12 & $8.94 \mathrm{E}-06$ & $4.02 \mathrm{E}-04$ \\
Regulation of actin cytoskeleton (K) & 0.021 & 214 & 10 & $1.35 \mathrm{E}-05$ & $5.54 \mathrm{E}-04$ \\
Signaling by SCF-KIT (R) & 0.0259 & 265 & 11 & $1.46 \mathrm{E}-05$ & $5.55 \mathrm{E}-04$ \\
Signaling by ERBB4 (R) & 0.0263 & 269 & 11 & $1.68 \mathrm{E}-05$ & $5.87 \mathrm{E}-04$ \\
NCAM signaling for neurite out-growth (R) & 0.0218 & 223 & 10 & $1.92 \mathrm{E}-05$ & $6.15 \mathrm{E}-04$ \\
Calcium signaling pathway (K) & 0.0175 & 179 & 9 & $2.13 \mathrm{E}-05$ & $6.38 \mathrm{E}-04$ \\
Signaling by NGF (R) & 0.0382 & 390 & 13 & $2.34 \mathrm{E}-05$ & $6.54 \mathrm{E}-04$ \\
RAF/MAP kinase cascade (R) & 0.0184 & 188 & 9 & $3.11 \mathrm{E}-05$ & $8.09 \mathrm{E}-04$ \\
Signaling by Type 1 Insulin-like Growth Factor 1 & 0.0236 & 241 & $3.69 \mathrm{E}-05$ & $9.23 \mathrm{E}-04$ \\
Receptor (IGF1R) (R) & & & & & \\
\hline
\end{tabular}

